\title{
Effects of Interval Training Under Hypoxia on the Autonomic Nervous System and Arterial and Hemorheological Function in Healthy Women
}

\author{
Hun-Young Park $\mathbb{D}^{1,2}$, Won-Sang Jung ${ }^{2}$, Sung-Woo Kim $\mathbb{D}^{2}$, Kiwon Lim ${ }^{1-3}$ \\ 'Department of Sports Medicine and Science, Graduated School, Konkuk University, Seoul, Republic of Korea; ${ }^{2}$ Physical Activity and Performance \\ Institute (PAPI), Konkuk University, Seoul, Republic of Korea; ${ }^{3}$ Department of Physical Education, Konkuk University, Seoul, Republic of Korea \\ Correspondence: Kiwon Lim, Department of Physical Education, Konkuk University, I20 Neungdong-ro, Gwangjin-gu, Seoul, 05029, Republic of \\ Korea, Tel +82-2-450-3827, Fax +82-2-450-3949, Email exercise@konkuk.ac.kr
}

\begin{abstract}
Purpose: The present study verified the effects of interval training under hypoxia, a novel exercise modality for health promotion, on the autonomic nervous system (ANS) and arterial and hemorheological function in healthy women.

Methods: Twenty healthy Korean women (age: 19-29 [24.85 \pm 3.84$]$ years) were equally assigned to interval normoxic training (INT, $\mathrm{n}=10$; residing and training under normoxia) and interval hypoxic training (IHT, $\mathrm{n}=10$; residing under normoxia and training under $526 \mathrm{mmHg}$ hypobaric hypoxia) groups. All participants performed 90-min of training sessions composed of 15-min of warm-up, 60min of interval training, and 15-min of cool-down. The interval training sessions composed of 10 repetitions of interval exercise using a treadmill (5 min of exercise corresponding to $90-95 \%$ maximal heart rate [HR] and 1 min of rest). The training was performed 3 days per week for 6 weeks. All participants underwent body composition, HR variability, brachial-ankle pulse wave velocity, flowmediated dilation (FMD), red blood cell (RBC) deformability and aggregation, and maximal oxygen uptake $\left(\mathrm{VO}_{2}\right.$ max) measurements before and after training.

Results: There were no significant differences in body composition between the groups. The IHT group showed a significant improvement in the ANS function (root mean square of successive differences, high frequency, and low frequency/high frequency ratio), arterial stiffness, arterial endothelial function (FMD), hemorheological function (RBC deformability and aggregation), and aerobic performance $\left(\mathrm{VO}_{2} \max \right)$ compared with the INT (all $\left.p<0.05\right)$.
\end{abstract}

Conclusion: In comparison with the interval training under normoxia, the interval training under hypoxia is a novel and effective exercise modality for promoting aerobic performance with the ANS and arterial and hemorheological function in healthy women.

Keywords: interval training, hypoxia, heart rate variability, arterial stiffness, arterial endothelial function, erythrocyte deformability and aggregation, maximal oxygen uptake

\section{Introduction}

Exercise is one of the most important health promotion strategies and preventative measures against many chronic diseases. ${ }^{1}$ Regular exercise also helps prevent weight gain and increases personal well-being by improving bone and muscle health, brain and mental health, and cardiovascular and metabolic health. ${ }^{1-4}$ The most cost-effective strategy to enhance public health is to promote exercise and to recommend regular physical activity. ${ }^{2}$ However, a growing number of individuals worldwide currently do not meet their recommended physical activity goals. ${ }^{5}$ The most often cited barriers to exercise participation are lack of time and financial costs. ${ }^{6}$

Moderate-intensity continuous training (MICT), involving continuous exercise at a moderate intensity, without rest, for $0.5-1$ hours, is generally prescribed for health promotion, prevention, and treatment of cardiovascular and metabolic diseases. ${ }^{1,6,7}$ However, MICT is not an appropriate exercise modality to address time constraints, a major problem reported by physically inactive individuals. ${ }^{6,8}$ Recently, high-intensity interval training (HIIT), involving high-intensity exercise separated by periods of passive or active recovery at low intensity, induces similar or superior benefits on 
cardiovascular and metabolic health in healthy and clinical populations, with less time commitment. ${ }^{9,10}$ HIIT appears to provide intermittent periods of relatively intense exercise within a training session, is less time constrained than MICT, and offers enhanced aerobic performance and cardiovascular and metabolic health benefits. ${ }^{10-12}$ Therefore, HIIT is widely used today for various health promotion and disease prevention activities. ${ }^{1,6-8}$

Recently, hypoxia has been used to maximize the usefulness of exercise in cardiovascular and metabolic health. ${ }^{13-15}$ The combination of hypoxia and exercise triggers specific responses, not observed by a similar exercise under normoxia. ${ }^{13,15,16}$ In particular, high-intensity exercise training under hypoxia showed a greater specific adaptation such as enhanced oxygen delivery and utilization capacity, cardiopulmonary function, mitochondrial capacity, oxidative enzyme capacity, angiogenesis, and muscle buffering capacity compared with exercise training under normoxia. ${ }^{17-22}$ Additionally, hypoxic exercise imposes less mechanical stress on the locomotor system, while resulting in similar physiological stress, and achieving a higher cardiometabolic demand. ${ }^{23,24}$ Therefore, exercise intervention under hypoxia is a novel exercise modality used in general medical practice in many developed countries. ${ }^{1,13-16,23-25}$

However, most previous experimental studies on exercise intervention under hypoxia have focused on weight loss, hemodynamic function, pulmonary function, and metabolic health, and most researchers have verified the effects of MICT under hypoxia on health promotion, prevention, and treatment of diseases. ${ }^{14,16,26,27}$ Additionally, autonomic nervous system (ANS) function, arterial stiffness, and arterial endothelial function are important determinants of cardiovascular health. ${ }^{13,15,25}$ However, few previous studies have verified the efficacy of interval training with high intensity under hypoxia as an effective exercise modality on ANS function, arterial stiffness, and arterial endothelial function.

Therefore, the present study aimed to investigate the effects of a 6-week interval training under moderate hypoxia (hypobaric hypoxic condition, $526 \mathrm{mmHg}$, a 3000-m simulated altitude) vs normoxia (normoxic condition, $760 \mathrm{mmHg}$ ) on the ANS and arterial and hemorheological function, and aerobic performance in healthy women. Our hypothesis is that HIIT under hypoxia vs normoxia may result in greater improvements in aerobic performance with ANS and arterial and hemorheological function in healthy women.

\section{Methods}

\section{Participants}

To first verify the safety and efficacy of exercise intervention under hypoxia, we selected 20 healthy Korean women (age: $19-29[24.85 \pm 3.84]$ years) who were non-smokers and non-overweight/obese and had no history of hypertension, diabetes, hyperlipidemia, cardiovascular, pulmonary or musculoskeletal diseases. They were equally assigned to an interval normoxic training (INT) or an interval hypoxic training (IHT) according to their body composition and aerobic performance. Participants were explained the purpose and process of the study, including possible side effects, and consent was obtained. All participants completed the study and the data were analyzed. To determine the sample size, we focused on identifying meaningful differences in flow-mediated dilation (FMD), as previously suggested. ${ }^{25}$ We set alpha to 0.05 and power to be 0.8 for sample size selection, and the total required sample size was estimated 16 participants ( 8 participants per group). The sample size was calculated using $G^{*}$ Power software (version 3.1.9.7, University of Kiel, Kiel, Germany). The consolidated standards of reporting trial (CONSORT: Consolidated Standards of Reporting Trials) flow diagram is shown in Figure 1 and characteristics of the subjects are presented in Table 1. The study was conducted in accordance with the provisions of the Declaration of Helsinki and was approved by the Institutional Review Board of Konkuk University (7001355-2020002-HR-359). The trial information is registered with the Clinical Research Information Service in Korea (КСТ0004517).

\section{Study Design}

The present study design is illustrated in Figure 2 and was designed similar to that of Jung et al. ${ }^{28}$ Participants were equally divided into INT ( $\mathrm{n}=10$, interval training under normoxia; $760 \mathrm{mmHg}$; sea level) and IHT $(\mathrm{n}=10$, interval training under hypoxia; $3000 \mathrm{~m}$ simulated altitude; $526 \mathrm{mmHg}$ ) groups. All testing procedures and training sessions were 


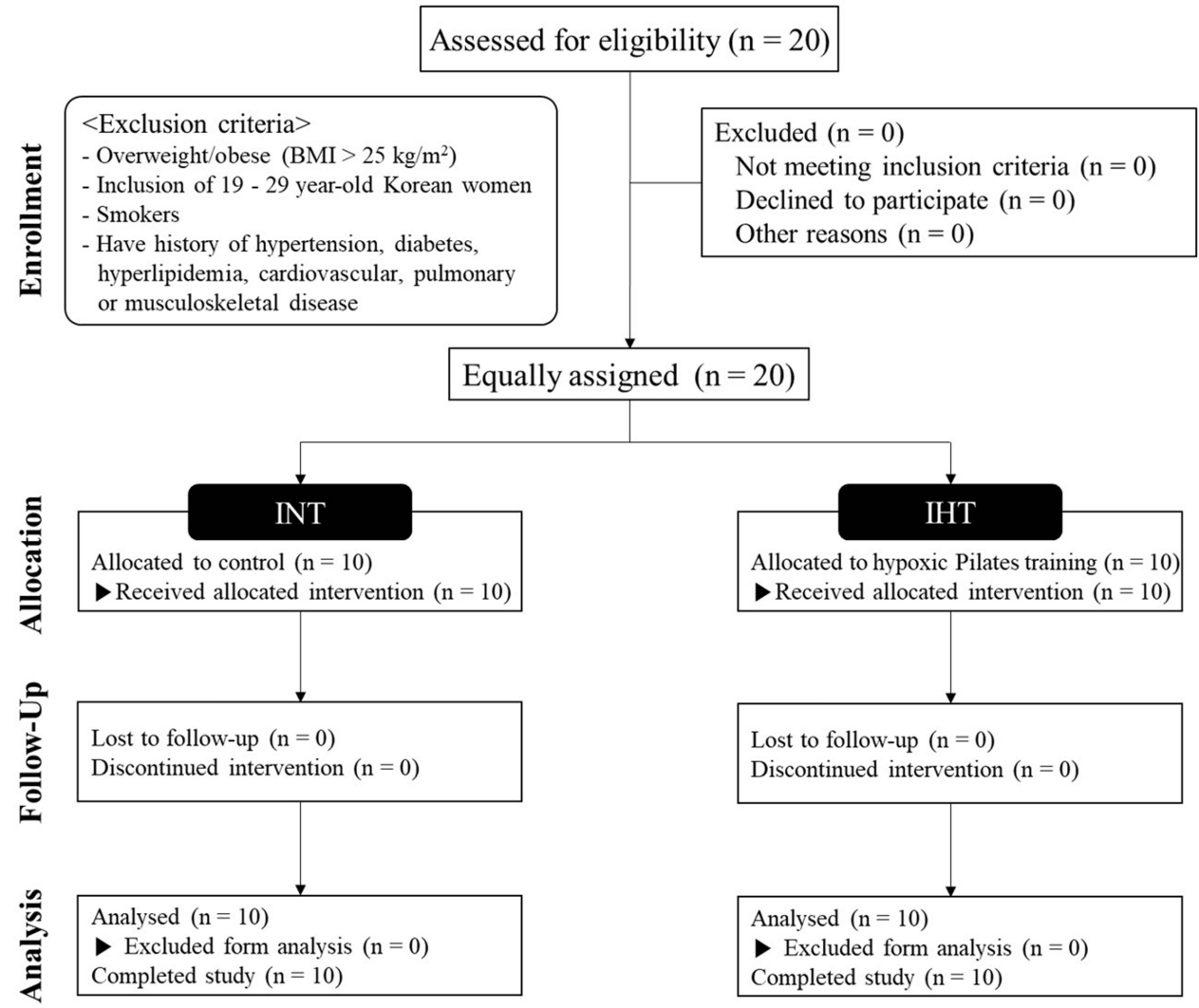

Figure I CONSORT (Consolidated Standards of Reporting Trials) flow diagram.

Abbreviations: INT, interval normoxic training; IHT, interval hypoxic training; BMI, body mass index.

performed in a 9-m (width) $\times 7-\mathrm{m}$ (length) $\times 3-\mathrm{m}$ (high) chamber at a temperature of $22 \pm 1{ }^{\circ} \mathrm{C}$ and humidity of $50 \pm 5 \%$, regulated by an environmental control chamber (NCTC-1, Nara control, Seoul, Republic of Korea).

We included a 3-day pre-testing period (eg, two testing days and one resting day between the testing days), a 6-week interval training session period under each environmental condition, and 3-day post-testing period (eg, two testing days and one resting day between the testing days). The post-test period began 3 days after the last training session.

Table I Characteristics of the Participants

\begin{tabular}{|c|c|c|c|c|}
\hline Variables & INT & IHT & $t$-value & $p$-value \\
\hline Number (n) & $\mathrm{n}=10$ & $\mathrm{n}=10$ & - & - \\
\hline Environmental condition $(\mathrm{mmHg})$ & Sea level $(760 \mathrm{mmHg})$ & $3000-\mathrm{m}$ simulated altitude $(526 \mathrm{mmHg})$ & - & - \\
\hline Age (year) & $24.5 \pm 3.8$ & $25.2 \pm 4.0$ & -0.398 & 0.695 \\
\hline Height (cm) & $164.8 \pm 0.9$ & $163.2 \pm 3.9$ & 1.243 & 0.243 \\
\hline Weight (kg) & $52.0 \pm 3.4$ & $51.2 \pm 4.7$ & 0.409 & 0.687 \\
\hline BMI $\left(\mathrm{kg} / \mathrm{m}^{2}\right)$ & $19.1 \pm 1.2$ & $19.2 \pm 1.6$ & -0.140 & 0.890 \\
\hline FFM (kg) & $35.6 \pm 2.3$ & $35.5 \pm 3.2$ & 0.057 & 0.955 \\
\hline Percent body fat (\%) & $20.7 \pm 0.9$ & $20.6 \pm 1.1$ & 0.090 & 0.929 \\
\hline SBP $(\mathrm{mmHg})$ & $108.9 \pm 11.0$ & $108.2 \pm 10.6$ & 0.145 & 0.886 \\
\hline DBP (mmHg) & $66.1 \pm 8.6$ & $65.3 \pm 7.9$ & 0.217 & 0.831 \\
\hline $\mathrm{VO}_{2} \max (\mathrm{mL} / \mathrm{kg} / \mathrm{min})$ & $49.7 \pm 4.6$ & $49.4 \pm 4.3$ & 0.131 & 0.897 \\
\hline
\end{tabular}

Note: Values are expressed as the mean \pm standard deviation.

Abbreviations: INT, interval normoxic training; IHT, interval hypoxic training; BMI, body mass index; FFM, free fat mass; SBP, systolic blood pressure; DBP, diastolic blood pressure. 

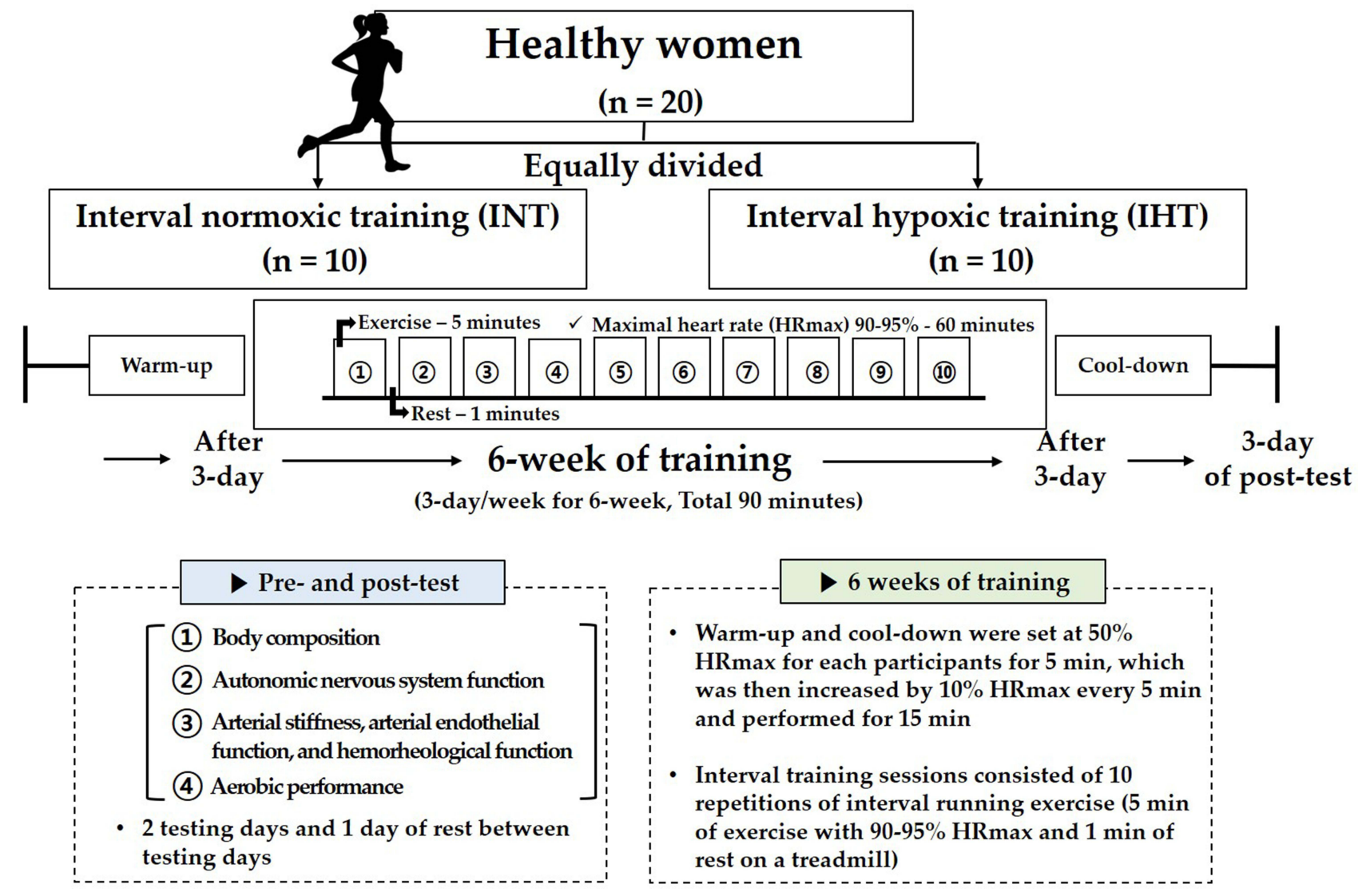

Figure 2 Study design.

On the first pre- and post-testing days, participants fasted for $>8 \mathrm{~h}$ and after stabilization, a venous blood sample was collected between 8:00 and 9:00 am to analyze hemorheological function parameters. Thereafter, body composition and ANS function were measured. On the second pre- and post-testing days, pulse wave velocity (PWV) and FMD, which are indicators of arterial function, were measured in order in the morning (between 7:00 and 11:00 am) after fasting for $>8 \mathrm{~h}$. After sufficient rest and meals, the maximal oxygen uptake $\left(\mathrm{VO}_{2} \mathrm{max}\right)$ was measured to evaluate aerobic performance.

During the interval training session period, participants performed the 90-min training sessions composed of warmup, interval training, and cool-down in their respective environmental conditions (INT: normoxic condition, $760 \mathrm{mmHg}$; IHT: moderate hypobaric hypoxic condition, $526 \mathrm{mmHg}$, a 3000-m simulated altitude). The training was performed 3 days per week for 6 weeks. Warm-up and cool-down were performed for 15 minutes, and all participants started with $50 \%$ HRmax and then increased by $10 \%$ HRmax every 5 minutes. The interval training sessions comprised 10 repetitions of interval exercise ( 5 min of exercise corresponding to $90-95 \%$ HRmax and 1 min of rest) on a treadmill. ${ }^{28}$ The running velocity on a treadmill was changed depending on the heart rate monitor (Polar M400, Helsinki, Finland) to match the 90-95\% HRmax.

Participants underwent body composition (height, weight, body mass index [BMI], free fat mass [FFM], and percent body fat), ANS function (root mean square of successive differences; RMSSD, low frequency; LF, high frequency; HF, and LF/HF ratio), arterial stiffness (brachial-ankle pulse wave velocity; baPWV), arterial endothelial function (PWV), hemorheological function (red blood cell $[\mathrm{RBC}]$ deformability and aggregation), and aerobic performance (VO ${ }_{2} \mathrm{max}$ ) measurements before and after the 6-week interval training session under each environmental condition.

\section{Body Composition}

Measurement of body composition was conducted based on previous studies. ${ }^{20}$ Body composition parameters (height, weight, BMI, FFM, and percent body fat) of the participants were analyzed using a stadiometer (YM-1, KDS, Seoul, 
Korea) and bioelectrical impedance analyzer (Inbody 770; Inbody, Seoul, Korea). Participants wore lightweight clothing and any metal items on their bodies were discarded.

\section{ANS Function}

Measurement of ANS function was conducted based on previous studies. ${ }^{16}$ Heart rate variability (HRV) was measured to examine changes in ANS function before and after the 6-week interval training in each environmental condition. HRV was measured by the following procedure: After taking a break for 10 minutes for stable measurement, 4 pads were attached to the wrist and ankle using an HRV meter (LAXTHA; CANS-3000, Daejeon, Korea), and HRV was measured in a sitting position with a steady state. Among the HRV parameters, RMSSD was analyzed as a time-domain method, and LF, HF, and LF/HF ratio were measured using the frequency-domain method. ${ }^{16,28}$

\section{Arterial Stiffness, Arterial Endothelial Function, and Hemorheological Function}

Measurement of arterial stiffness, arterial endothelial function, and hemorheological function was conducted based on previous studies. ${ }^{24,25}$ The baPWV, as a parameter of arterial stiffness, was verified using an automatic oscillometric device (VP-1000 plus, Omron, Osaka, Japan). The VP-1000 plus simultaneously records the baPWV and brachial and ankle BPs on the left and right sides, generates an electrocardiogram, and records heart sounds. Electrocardiography electrodes were placed bilaterally on both wrists and cuffs. A microphone for detecting heart sounds was placed on the left edge of the sternum. The cuffs were connected to a plethysmographic sensor, which determined the volume pulse form and an oscillometric pressure sensor that measured BP. The brachial and ankle pulse volume waveforms were recorded using a semiconductor pressure sensor. The baPWV values on the right and left sides were obtained and averaged for analysis.

The FMD in the right brachial artery, as an arterial endothelial indicator, was measured via noninvasive Doppler ultrasound (UNEX-EF, Tokyo, Japan); we fixed the instrument in the brachial artery region 3-5 cm above the elbow and measured the diameter of the medial muscle artery. Next, blood was removed for $5 \mathrm{~min}$ by increasing BP by $50 \mathrm{mmHg}$ from the resting BP. After 5 min, deflation was automatically recorded for the next 2 min, and the calculated values of FMD (FMD $=[$ reactive hyperemia diameter - baseline diameter $] \times 100 \%$ ) were used to evaluate the diameter and blood flow rate.

Participants were evaluated for RBC deformability and aggregation as hemorheological function parameters to evaluate microvascular circulation function. We analyzed RBC deformability and aggregation using Rheoscan-D under environmental conditions of $25^{\circ} \mathrm{C}$ and $3 \mathrm{~Pa}$ shear stress within $4-6 \mathrm{~h}$ after blood collection. ${ }^{25,29} \mathrm{RBC}$ deformability was measured using the elongation index (EI), wherein we first transferred the sample to a 2-mL microseparation tube, which was diluted in $700 \mu \mathrm{L}$ of $5.5 \%$ polyvinylpyrrolidone ( $360 \mathrm{kDa}$ ) dissolved in $1 \mathrm{mmol}$ phosphate-buffered saline (pH 7.4; osmolality: $300 \mathrm{mOsmol} /$ $\mathrm{kg}$ ) in a K3-ethylenediaminetetraacetic acid tube (Greiner Bio-one, Chon Nuri, Thailand). Thereafter, this solution $(0.5 \mathrm{~mL})$ was analyzed using a D-test kit according to Rheo Meditech Inc.'s instructions. The accuracy of the RBC EI was measured using a Lineweaver-Burk plot model. ${ }^{30} \mathrm{RBC}$ aggregation was measured using the aggregation index; we analyzed $8 \mu \mathrm{L}$ of the whole blood sample using an A-test kit according to Rheo Meditech Inc.'s instructions. ${ }^{31}$

\section{Aerobic Performance}

Measurement of aerobic performance was conducted based on previous studies. ${ }^{28} \mathrm{VO}_{2}$ max was measured as an aerobic performance parameter before and after interval training in each environmental condition using the modified Bruce protocol for graded exercise testing on a treadmill (S25TX, SFET, Seoul, Republic of Korea) using a K5 breath-by-breath auto metabolism analyzer (K5, Cosmed, Rome, Italy). The graded exercise test was completed when the following three criteria were satisfied: 1) $\mathrm{VO}_{2}$ plateau - no further increase in oxygen use per minute even with an increase in work performed, 2) HR within 10 beats of the age-predicted HRmax, which is the basis for using the participants' HRmax as a surrogate for the $\mathrm{VO}_{2} \max$ when designing personal training programs, 3) respiratory exchange ratio $>1.5$, and 4) plasma (blood) lactate concentrations $>7 \mathrm{mmol} / \mathrm{L}$.

\section{Statistical Analysis}

The means and stDLFandard deviations were calculated for each primary dependent parameter. The normality of the distribution of all outcome parameters was verified using the Shapiro-Wilk W-test prior to parametric tests. A two-way 
analysis of variance ("time" $\times$ "group") with repeated measures of the "time" factor was used to analyze the effects of the interval training environmental condition on each dependent variable. Partial eta-squared $\left(\eta^{2}\right)$ values were calculated as measures of the effect size. If a significant interaction was found, a Bonferroni post-hoc test was performed to identify within-group changes over time. Additionally, a paired $t$-test was performed to compare the post-training versus pretraining values of dependent parameters in each group separately. We used Cohen's d (effect size), which reflects the value of a statistic calculated from a sample of data and standardized mean differences. Statistical differences in the means (effect size, Cohen's d) were determined with a significance level $(p<0.05)$ and 95\% confidence interval (CI). All analyses were performed using SPSS Statistics (version 25.0; IBM Corp., Armonk, NY, USA).

\section{Results}

Body composition data of the INT and IHT groups before and after training are presented in Table 2. No significant interaction was observed for any of the body composition parameters; the body composition did not affect changes in the ANS function, arterial stiffness, endothelial function, hemorheological function, and aerobic performance.

Figure 3 depicts the pre- and post-training ANS function data via the measured HRV. There was a significant interaction in $\operatorname{RMSSD}\left(\eta^{2}=0.449, p=0.001\right)$, HF $\left(\eta^{2}=0.346, p=0.006\right)$, and LF/HF ratio $\left(\eta^{2}=0.461, p=0.001\right)$; however, no significant interaction was noted for LF. In the post-hoc analysis, RMSSD (Cohen's d: $1.22,95 \%$ CI: 0.25 , $2.08, p<0.05$ ) and HF (Cohen's d: $0.35,95 \%$ CI: $-0.51,1.18, p<0.05$ ) showed a significant increase in the IHT. The LF/HF ratio showed a significant increase in the INT (Cohen's d: $0.67,95 \% \mathrm{CI}:-0.22,1.50, p<0.05$ ), but a significant decrease in the IHT (Cohen's d: $-0.77,95 \% \mathrm{CI}:-1.61,0.13, p<0.05$ ).

Arterial stiffness, arterial endothelial function, and hemorheological function are shown in Figure 4, and a significant interaction was found for baPWV $\left(\eta^{2}=0.348, p=0.006\right)$, FMD $\left(\eta^{2}=0.428, p=0.002\right)$, RBC deformability $\left(\eta^{2}=0.501\right.$, $p<0.001$ ), and RBC aggregation $\left(\eta^{2}=0.410, p=0.002\right)$. In the post-hoc analysis, baPWV (Cohen's d: $-1.10,95 \%$ CI: $-1.95,-0.15, p<0.05$ ) and RBC deformability (Cohen's d: $2.34,95 \%$ CI: $1.15,3.33, p<0.05$ ) showed a significant increase in the IHT. Additionally, FMD showed a significant increase (INT = Cohen's d: 1.77, 95\% CI: 0.70, 2.68, $p<$ 0.05 , IHT $=$ Cohen's d: $3.71,95 \%$ CI: $2.17,4.94, p<0.05)$ and RBC aggregation showed a significant decrease $(\mathrm{INT}=$ Cohen's d: $-0.07,95 \% \mathrm{CI}:-0.91,0.77, p<0.05$; IHT = Cohen's d: $-0.31,95 \% \mathrm{CI}:-1.15,0.54, p<0.05)$ in both groups. However, a greater improvement in the FMD and RBC aggregation was observed in the IHT group than in the INT group.

The aerobic performance of both groups is shown in Figure 5. There was a significant interaction in the $\mathrm{VO}_{2} \max \left(\eta^{2}\right.$ $=0.237, p=0.037$ ). Post-hoc analyses revealed a significant increase in the $\mathrm{VO}_{2} \max$ in the INT (Cohen's d: $0.94,95 \%$ CI: $0.02,1.79, p<0.05$ ) and IHT (Cohen's d: $1.15,95 \%$ CI: $0.19,0.00, p<0.05$ ), but a greater improvement in $\mathrm{VO}_{2}$ max was observed in the IHT group than in the INT group.

\section{Discussion}

Our study demonstrated that the IHT is effective in improving ANS (eg, RMSSD, HF, and LF/HF ratio) and arterial (eg, baPWV and FMD) and hemorheological function (eg, RBC deformability and aggregation) compared with INT in healthy women.

Regarding body composition, previous studies have reported that exercise intervention under hypoxia is effective in reducing weight and body fat via improved cardiometabolic function in overweight/obese population. ${ }^{26,27,32}$ Wiesner et $\mathrm{al}^{27}$ conducted a study in overweight to obese subjects to test the hypothesis that 4 weeks of MICT under hypoxia $(\mathrm{n}=$ $\left.24, \mathrm{~F}_{\mathrm{i}} \mathrm{O}_{2}=15 \%\right)$ results in similar or even greater improvement in body weight and metabolic risk markers compared with exercise under normoxia $\left(\mathrm{n}=21, \mathrm{FiO}_{2}=21 \%\right)$. They reported that the hypoxic group showed a greater weight loss and similar physical fitness and metabolic risk markers at lower workloads compared with the normoxic group. Kong et $\mathrm{al}^{26}$ investigated the additive effect of 4 weeks of MICT under hypoxia $\left(\mathrm{n}=11, \mathrm{~F}_{\mathrm{i}} \mathrm{O}_{2}=16.4-14.5 \%\right)$ vs normoxia $(\mathrm{n}=$ $11, \mathrm{~F}_{\mathrm{i}} \mathrm{O}_{2}=21 \%$ ) on weight loss in obese young adults, and concluded that hypoxic training resulted in an additive improvement in weight loss. Hobbins et $\mathrm{al}^{32}$ conducted a systematic review to synthesize the effects of exercise intervention under hypoxia on body composition and cardiometabolic health. They concluded that exercise intervention under hypoxia resulted in increased energy expenditure, decreased weight and body fat, and improved cardiometabolic 
Table 2 Changes in Body Composition Before and After Training at Each Environmental Condition

\begin{tabular}{|c|c|c|c|c|c|c|c|c|c|}
\hline \multirow[t]{2}{*}{ Variables } & \multicolumn{2}{|c|}{ INT $(n=10)$} & \multirow{2}{*}{$\begin{array}{l}\text { Cohen's d } \\
(95 \% \mathrm{CI})\end{array}$} & \multicolumn{2}{|c|}{ IHT $(n=10)$} & \multirow{2}{*}{$\begin{array}{l}\text { Cohen's d } \\
(95 \% \mathrm{Cl})\end{array}$} & \multicolumn{3}{|c|}{$p$-value $\left(\eta^{2}\right)$} \\
\hline & Pre & Post & & Pre & Post & & Time & Group & Interaction \\
\hline Weight (kg) & $52.0 \pm 3.4$ & $51.7 \pm 3.3$ & $-0.07(-0.90,0.78)$ & $51.2 \pm 4.7$ & $51.1 \pm 4.4$ & $-0.03(-0.87,0.8 \mathrm{I})$ & $0.057(0.187)$ & $0.697(0.009)$ & $0.659(0.011)$ \\
\hline BMI $\left(\mathrm{kg} / \mathrm{m}^{2}\right)$ & $19.1 \pm 1.2$ & $19.0 \pm 1.1$ & $-0.07(-0.91,0.77)$ & $19.2 \pm 1.6$ & $19.2 \pm 1.6$ & $0.00(-0.83,0.84)$ & $0.191(0.093)$ & $0.829(0.003)$ & $0.119(0.130)$ \\
\hline FFM (kg) & $35.6 \pm 2.3$ & $35.4 \pm 2.4$ & $-0.07(-0.90,0.78)$ & $35.5 \pm 3.2$ & $35.5 \pm 3.2$ & $0.00(-0.84,0.84)$ & $0.539(0.021)$ & $1.000(0.000)$ & $0.590(0.016)$ \\
\hline Percent body fat (\%) & $20.7 \pm 0.9$ & $20.6 \pm 0.8$ & $-0.09(-0.93,0.75)$ & $20.6 \pm 1.1$ & $20.4 \pm 1.4$ & $-0.15(-0.99,0.70)$ & $0.150(0.112)$ & $0.806(0.003)$ & $0.476(0.029)$ \\
\hline
\end{tabular}

Note: Values are expressed as the mean \pm standard deviation.

Abbreviations: INT, interval normoxic training; IHT, interval hypoxic training; BMI, body mass index; FFM, free fat mass; $\mathrm{Cl}$, confidence interval. 

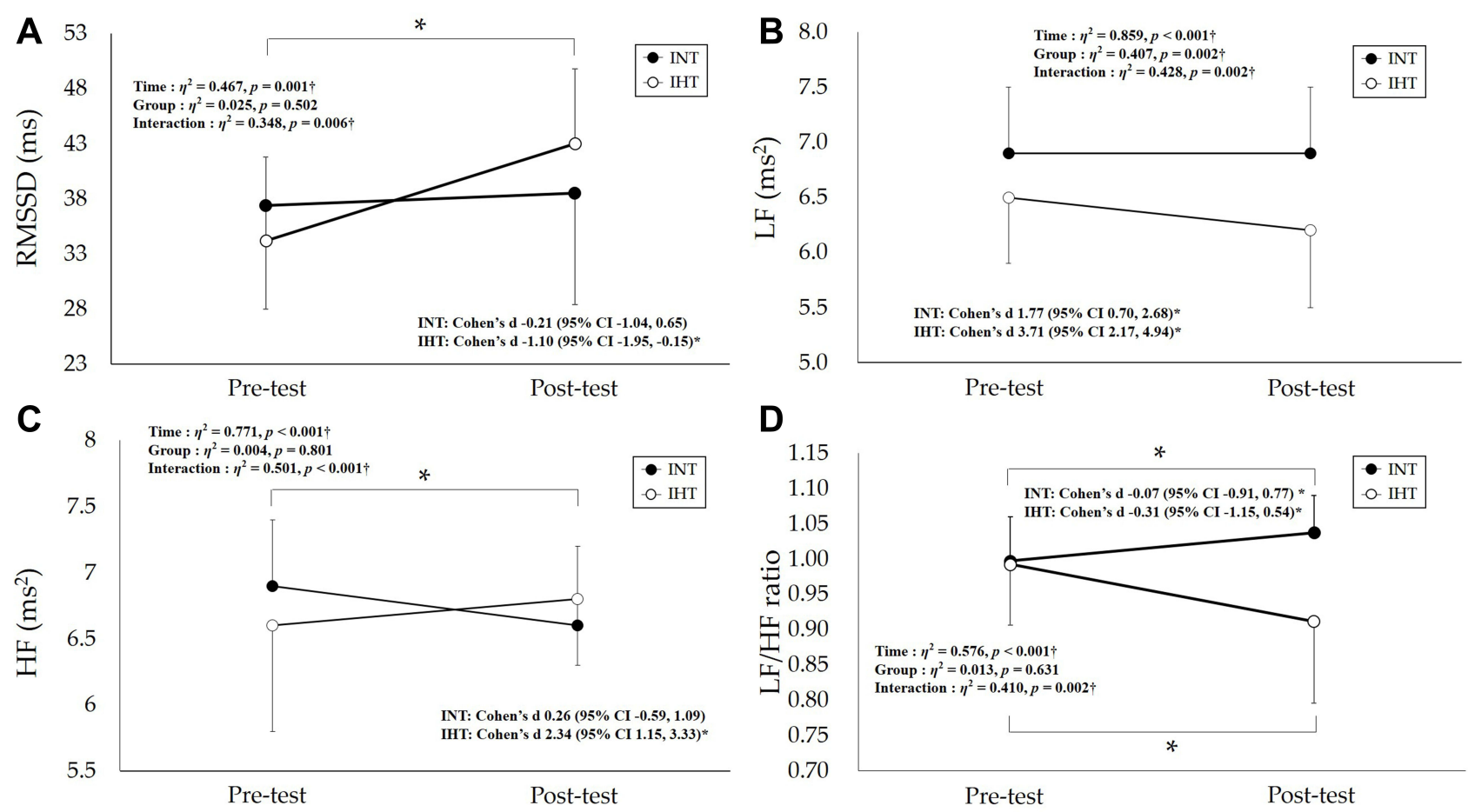

Figure 3 Changes in ANS function before and after training at each environmental condition. (A) Change in the RMSSD. (B) Change in LF. (C) Change in HF. (D) Change in LF/HF ratio. ${ }^{\dagger} p<0.05$, significant interaction or main effect. ${ }^{*} p<0.05$, significant difference between before and after training at each environmental condition.

Abbreviations: RMSSD, root mean square of successive differences; LF, low frequency; HF, high frequency; ANS, autonomic nervous system; INT, interval normoxic training; IHT, interval hypoxic training; $\mathrm{Cl}$, confidential interval.

health in the obese population. However, our study did not show any change in body composition parameters, such as weight and body fat, after 6 weeks of exercise training session as all participants were healthy women with normal range of body composition (BMI $20.7 \pm 1.3$ and \%body fat 20.9 \pm 1.1 ). Our results are considered reasonable in that there was no body composition change in comparison to results from previous studies that conducted various hypoxic training on athletes or in the healthy population. ${ }^{17-21}$

HRV reflects the interactions of the sympathetic nervous system (SNS) and parasympathetic nervous system (PNS), which modulate cardiovascular function, and is used to evaluate ANS function. ${ }^{16,28,33}$ In particular, HRV is widely used to examine changes in ANS activity and balance due to stress and to obtain information on stress-related diseases. ${ }^{16,28,33}$ Regarding HRV parameters, RMSSD is mainly used to predict HRV at high frequencies and mainly reflects the activity of the PNS. ${ }^{28,34}$ A higher RMSSD is interpreted as a physiologically healthy and relaxed state. ${ }^{28,34} \mathrm{HF}$ mainly reflects the activity of the vagus nerve branching into the heart and is a representative measure of PNS activity. ${ }^{16,28}$ LF is related to mental stress caused by SNS activation and indicates heart instability. ${ }^{28,33}$ Additionally, LF/HF reflects the overall balance of the ANS, and a higher value indicates that the SNS is relatively enhanced or that the PNS is suppressed. ${ }^{16,33}$ Few previous studies have been conducted on exercise training under hypoxia vs normoxia on changes in ANS function measured based on HRV. Park et $\mathrm{al}^{16}$ examined the effect of a 12-week combined exercise intervention under hypoxia (n $\left.=12, \mathrm{FiO}_{2}=14.5 \%\right)$ vs normoxia $\left(\mathrm{n}=12, \mathrm{FiO}_{2}=20.9 \%\right)$ on $\mathrm{HRV}$ in an obese elderly population. They reported that hypoxic training resulted in an improved $\mathrm{HF}$ and LF compared to normoxic training. Jung et al ${ }^{28}$ evaluated the effects of a 6-week IHT $\left(\mathrm{n}=10, \mathrm{FiO}_{2}=14.5 \%\right)$ vs INT $\left(\mathrm{n}=10, \mathrm{FiO}_{2}=20.9 \%\right)$ on ANS function in middle- and long-distance runners. They demonstrated that IHT enhances ANS function by improving RMSSD, HF, and LF/HF. Our study also verified that the IHT is an effective exercise modality that enhances ANS function via RMSSD, HF, and LF/HF compared with INT. In addition, Kim et al ${ }^{35}$ investigated whether six weeks of aerobic continuous and interval training under hypoxia $(\mathrm{n}=10,526 \mathrm{mmHg}$, simulated $3000 \mathrm{~m})$ can improve ANS function and aerobic performance versus normoxia $(\mathrm{n}=12,760 \mathrm{mmHg})$ in amateur male swimmers. They reported that hypoxic training composed of aerobic continuous and interval exercise improves the aerobic performance of amateur male swimmers with better ANS function. 

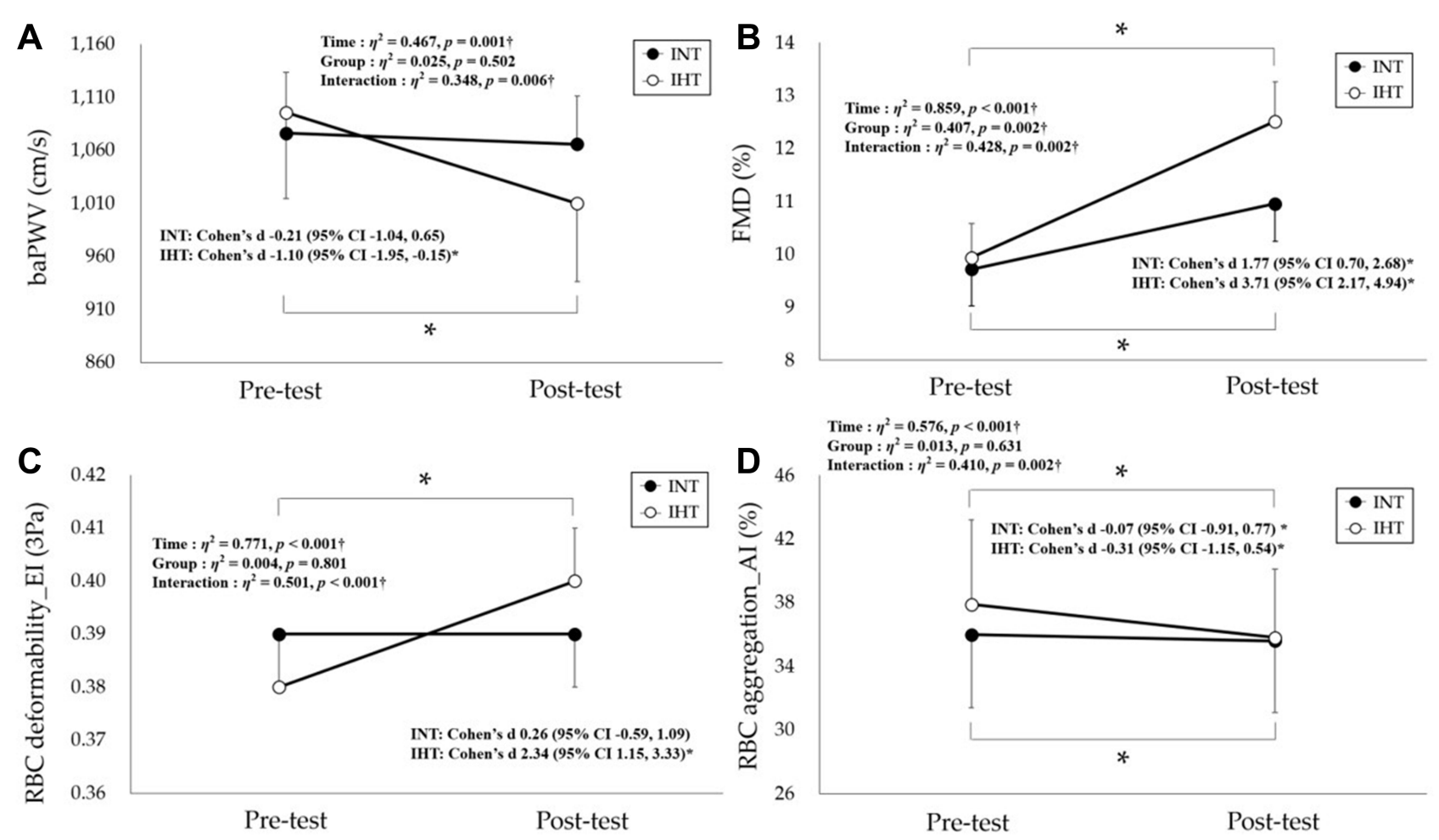

Figure 4 Changes in arterial stiffness, arterial endothelial function, and hemorheological function before and after training at each environmental condition. (A) Change in baPWV. (B) Change in FMD. (C) Change in RBC deformability. (D) Change in RBC aggregation. ${ }^{\dagger} p<0.05$, significant interaction or main effect. ${ }^{*} p<0.05$, significant difference before and after training at each environmental condition.

Abbreviations: baPWV, brachial-ankle pulse wave velocity; FMD, flow-mediated dilation; RBC, red blood cell; El, elongation index; Al, aggregation index; INT, interval normoxic training; IHT, interval hypoxic training; $\mathrm{Cl}$, confidential interval.

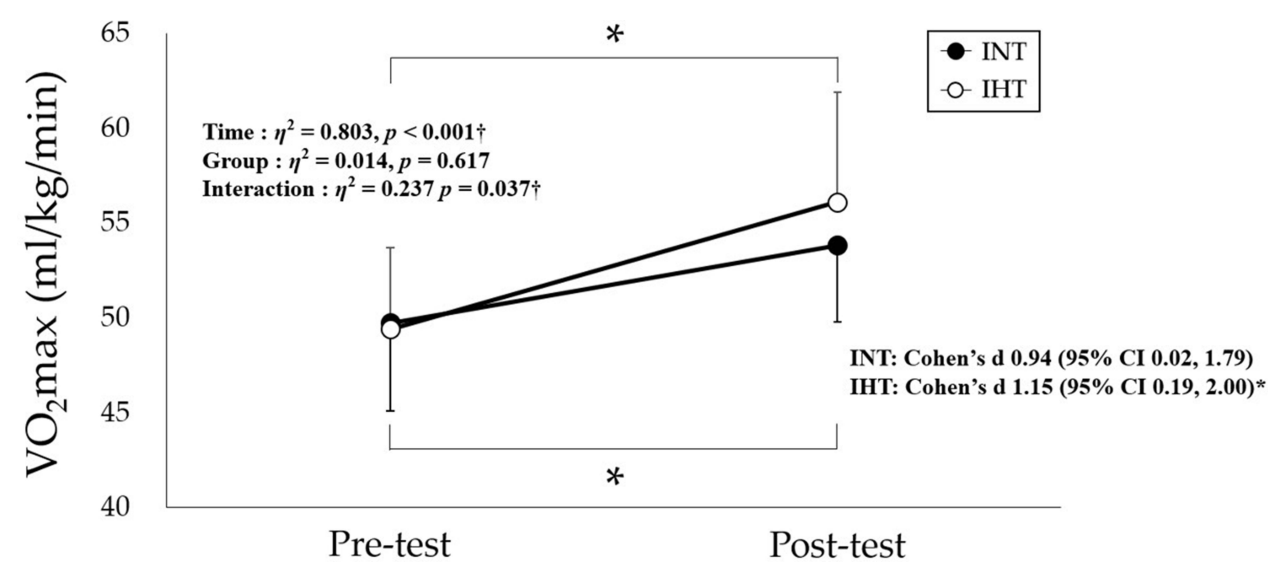

Figure 5 Changes in $\mathrm{VO}_{2}$ max before and after training at each environmental condition. ${ }^{\dagger} p<0.05$, significant interaction or main effect. ${ }^{*} p<0.05$, significant difference before and after training at each environmental condition.

Abbreviations: $\mathrm{VO}_{2} \mathrm{max}$, maximal oxygen uptake; INT, interval normoxic training; IHT, interval hypoxic training; $\mathrm{Cl}$, confidential interval.

These results suggest that exercise training under hypoxia increases the vagus nerve activity, thereby inhibiting the activity of the SNS compared with exercise training under normoxia.

Regarding arterial and hemorheological functions, both exercise and hypoxia showed an outstanding improvement. Regular exercise intervention increases vasodilation and upregulates endothelial nitric oxide (NO) synthases, which in turn induce augmented blood flow. ${ }^{35}$ Hypoxia induces an improvement in the bioavailability of NO, a major vasodilator, thereby enhancing vasodilation and reducing total peripheral resistance and reducing arterial stiffness and blood 
pressure. ${ }^{13,24,25,37}$ In particular, previous studies reported that exercise training under hypoxia promotes microcirculatory function for oxygen and carbon dioxide exchange in tissues and leads to improved ANS function, hemodynamic function, arterial stiffness, and vasodilation. ${ }^{13,24,25,37}$ Nishiwaki et al ${ }^{38}$ examined the effect of an 8-week exercise intervention under hypoxia $\left(\mathrm{n}=8, \mathrm{FiO}_{2}=16.5 \%\right)$ vs normoxia $\left(\mathrm{n}=8, \mathrm{FiO}_{2}=20.9 \%\right)$ on arterial stiffness and arterial endothelial function in postmenopausal women, reporting that hypoxic training may induce vascular functional adaptations with an increase in FMD response. Jung et $\mathrm{al}^{25}$ investigated the effect of a 12-week Pilates exercise under hypoxia $\left(\mathrm{n}=12, \mathrm{FiO}_{2}=14.5 \%\right)$ vs normoxia $\left(\mathrm{n}=10, \mathrm{FiO}_{2}=20.9 \%\right)$ on blood pressure, arterial stiffness, arterial endothelial function, and hemorheological function in obese women. They reported that hypoxic training induced an improvement in diastolic blood pressure, flow-mediated dilation, and erythrocyte deformability and aggregation compared with normoxic training and concluded that exercise intervention under hypoxia is a novel and successful modality for promoting arterial endothelial and hemorheological function. Based on these previous studies, we analyzed baPWV, FMD, and RBC deformability and aggregation before and after 6 weeks of IHT in healthy women. We confirmed that IHT is an effective exercise modality for improving arterial stiffness, arterial endothelial function, and hemorheological function compared with INT. Overall, our findings on arterial and hemorheological function via IHT are consistent with those of previous studies.

Aerobic performance reflects the ability of the cardiopulmonary system and microcirculatory ability to transport oxygen to muscle tissues during exercise and is an important indicator of metabolic and cardiovascular health. ${ }^{39,40}$ Exercise training under hypoxia enhances aerobic performance not only via erythropoiesis, but also via various factors such as the muscular nervous system, metabolic hormones, and various proteins that change muscle tissue very efficiently in oxygen and energy utilization. ${ }^{20-22,28}$ In particular, hypoxic training enhances aerobic performance via improved exercise economy, blood supply and utilization to tissues, capillary and mitochondrial density, oxidative and glycolytic enzyme activity, glucose transport, and acid-base balance. ${ }^{20-22,28,41,42}$ Consistent with previous studies, we also found that a 6-week IHT enhanced $\mathrm{VO}_{2} \mathrm{max}$ in healthy women compared with INT, and the enhanced aerobic performance is presumably due to the improvement in ANS and arterial and hemorheological function.

\section{Limitations}

Our study has some limitations. The lack of assessment of dietary intake as well as small sample size and/or the low number of participants were limitations of our study.

\section{Conclusion}

Our study confirmed that a 6-week interval training under hypoxia (526 mmHg, a 3000-m simulated altitude) would enhance the ANS and the arterial and hemorheological function, and the aerobic performance in healthy women. However, there is a need to confirm effective hypoxic training methods by verifying the differences according to various types of exercise under hypoxia (eg, MICT or HIIT) in the future.

\section{Ethics Approval and Consent to Participate}

The present study was approved by the Institutional Review Board of Konkuk University (7001355-2020002-HR-359) and was conducted in accordance with the provisions of the Declaration of Helsinki. Written informed consent was obtained from all participants.

\section{Acknowledgments}

We are particularly grateful to all the people who provided assistance to our article.

\section{Author Contributions}

All authors made a significant contribution to the work reported, whether that is in the conception, study design, execution, acquisition of data, analysis and interpretation, or in all these areas; took part in drafting, revising or critically reviewing the article; gave final approval of the version to be published; have agreed on the journal to which the article has been submitted; and agree to be accountable for all aspects of the work. 


\section{Funding}

This work was supported by the Ministry of Education of the Republic of Korea and the National Research Foundation of Korea (NRF-2019S1A5B8099542). This study was also supported by the KU Research Professor Program of Konkuk University.

\section{Disclosure}

The authors declare no conflicts of interest.

\section{References}

1. Park HY, Jung WS, Kim J, Hwang H, Lim K. Changes in the paradigm of traditional exercise in obesity therapy and application of a new exercise modality: a narrative review article. Iran J Public Health. 2019;48(8):1395-1404.

2. Kachur S, Chongthammakun V, Lavie CJ, et al. Impact of cardiac rehabilitation and exercise training programs in coronary heart disease. Prog Cardiovasc Dis. 2017;60(1):103-114. doi:10.1016/j.pcad.2017.07.002

3. Fletcher GF, Landolfo C, Niebauer J, Ozemek C, Arena R, Lavie CJ. Promoting physical activity and exercise: JACC health promotion series. J Am Coll Cardiol. 2018;72(14):1622-1639. doi:10.1016/j.jacc.2018.08.2141

4. Kim SW, Jung SW, Seo MW, Park HY, Song JK. Effects of bone-specific physical activity on body composition, bone mineral density, and health-related physical fitness in middle-aged women. J Exerc Nutrition Biochem. 2019;23(4):36-42. doi:10.20463/jenb.2019.0030

5. Rowe GC, Safdar A, Arany Z. Running forward: new frontiers in endurance exercise biology. Circulation. 2014;129(7):798-810. doi:10.1161/ CIRCULATIONAHA.113.001590

6. Gripp F, Nava RC, Cassilhas RC, et al. HIIT is superior than MICT on cardiometabolic health during training and detraining. Eur J Appl Physiol. 2021;121(1):159-172. doi:10.1007/s00421-020-04502-6

7. Su L, Fu J, Sun S, et al. Effects of HIIT and MICT on cardiovascular risk factors in adults with overweight and/or obesity: a meta-analysis. PLoS One. 2019;14(1):e0210644. doi:10.1371/journal.pone.0210644

8. Wewege M, van den Berg R, Ward RE, Keech A. The effects of high-intensity interval training vs. moderate-intensity continuous training on body composition in overweight and obese adults: a systematic review and meta-analysis. Obes Rev. 2017;18(6):635-646. doi:10.1111/obr.12532

9. Gillen JB, Gibala MJ. Interval training: a time-efficient exercise strategy to improve cardiometabolic health. Appl Physiol Nutr Metab. 2018;43(10): iii-iv. doi:10.1139/apnm-2018-0453

10. Batacan RB Jr., Duncan MJ, Dalbo VJ, Tucker PS, Fenning AS. Effects of high-intensity interval training on cardiometabolic health: a systematic review and meta-analysis of intervention studies. Br J Sports Med. 2017;51(6):494-503. doi:10.1136/bjsports-2015-095841

11. Kessler HS, Sisson SB, Short KR. The potential for high-intensity interval training to reduce cardiometabolic disease risk. Sports Med. 2012;42 (6):489-509. doi:10.2165/11630910-000000000-00000

12. Jelleyman C, Yates T, O'Donovan G, et al. The effects of high-intensity interval training on glucose regulation and insulin resistance: a meta-analysis. Obes Rev. 2015;16(11):942-961. doi:10.1111/obr.12317

13. Millet GP, Debevec T, Brocherie F, Malatesta D, Girard O. Therapeutic use of exercising in hypoxia: promises and limitations. Front Physiol. 2016;7:224. doi:10.3389/fphys.2016.00224

14. Haufe S, Wiesner S, Engeli S, Luft FC, Jordan J. Influences of normobaric hypoxia training on metabolic risk markers in human subjects. Med Sci Sports Exerc. 2008;40(11):1939-1944. doi:10.1249/MSS.0b013e31817f1988

15. Park HY, Kim J, Park MY, et al. Exposure and exercise training in hypoxic conditions as a new obesity therapeutic modality: a mini review. $J$ Obes Metab Syndr. 2018;27(2):93-101. doi:10.7570/jomes.2018.27.2.93

16. Park HY, Jung WS, Kim J, Lim K. Twelve weeks of exercise modality in hypoxia enhances health-related function in obese older Korean men: a randomized controlled trial. Geriatr Gerontol Int. 2019;19(4):311-316. doi:10.1111/ggi.13625

17. Faiss R, Léger B, Vesin JM, et al. Significant molecular and systemic adaptations after repeated sprint training in hypoxia. PLoS One. 2013;8(2): e56522. doi:10.1371/journal.pone.0056522

18. Galvin HM, Cooke K, Sumners DP, Mileva KN, Bowtell JL. Repeated sprint training in normobaric hypoxia. Br J Sports Med. 2013;47(Suppl1): i74-i79. doi:10.1136/bjsports-2013-092826

19. Geiser J, Vogt M, Billeter R, Zuleger C, Belforti F, Hoppeler H. Training high-living low: changes of aerobic performance and muscle structure with training at simulated altitude. Int J Sports Med. 2001;22(8):579-585. doi:10.1055/s-2001-18521

20. Park HY, Lim K. Effects of hypoxic training versus normoxic training on exercise performance in competitive swimmers. J Sports Sci Med. 2017;16(4):480-488.

21. Park HY, Shin C, Lim K. Intermittent hypoxic training for 6 weeks in 3000 m hypobaric hypoxia conditions enhances exercise economy and aerobic exercise performance in moderately trained swimmers. Biol Sport. 2018;35(1):49-56. doi:10.5114/biolsport.2018.70751

22. Nam SS, Park HY. Effects of endurance exercise under hypoxia on acid-base and ion balance in healthy males. Phys Act Nutr. 2020;24(3):7-12. doi:10.20463/pan.2020.0015

23. Girard O, Malatesta D, Millet GP. Walking in hypoxia: an efficient treatment to lessen mechanical constraints and improve health in obese individuals? Front Physiol. 2017;8:73. doi:10.3389/fphys.2017.00073

24. Jung K, Seo J, Jung WS, Kim J, Park HY, Lim K. Effects of an acute pilates program under hypoxic conditions on vascular endothelial function in pilates participants: a randomized crossover trial. Int J Environ Res Public Health. 2020;17(7):2584.

25. Jung K, Kim J, Park HY, Jung WS, Lim K. Hypoxic pilates intervention for obesity: a randomized controlled trial. Int J Environ Res Public Health. 2020;17(19). doi:10.3390/ijerph17197186

26. Kong Z, Zang Y, Hu Y. Normobaric hypoxia training causes more weight loss than normoxia training after a 4-week residential camp for obese young adults. Sleep Breath. 2014;18(3):591-597. doi:10.1007/s11325-013-0922-4 
27. Wiesner S, Haufe S, Engeli S, et al. Influences of normobaric hypoxia training on physical fitness and metabolic risk markers in overweight to obese subjects. Obesity. 2010;18(1):116-120. doi:10.1038/oby.2009.193

28. Jung WS, Kim SW, Park HY. Interval hypoxic training enhances athletic performance and does not adversely affect immune function in middleand long-distance runners. Int J Environ Res Public Health. 2020;17(6):1934.

29. Uyuklu M, Cengiz M, Ulker P, et al. Effects of storage duration and temperature of human blood on red cell deformability and aggregation. Clin Hemorheol Microcirc. 2009;41(4):269-278. doi:10.3233/CH-2009-1178

30. Baskurt OK, Meiselman HJ. Analyzing shear stress-elongation index curves: comparison of two approaches to simplify data presentation. Clin Hemorheol Microcirc. 2004;31(1):23-30

31. Kim SW, Jung WS, Park W, Park HY. Twelve weeks of combined resistance and aerobic exercise improves cardiometabolic biomarkers and enhances red blood cell hemorheological function in obese older men: a randomized controlled trial. Int J Environ Res Public Health. $2019 ; 16: 24$. doi:10.3390/ijerph16245020

32. Hobbins L, Hunter S, Gaoua N, Girard O. Normobaric hypoxic conditioning to maximize weight loss and ameliorate cardio-metabolic health in obese populations: a systematic review. Am J Physiol Regul Integr Comp Physiol. 2017;313(3):R251-r264. doi:10.1152/ajpregu.00160.2017

33. Kim J, Park HY, Lim K. Effects of 12 weeks of combined exercise on heart rate variability and dynamic pulmonary function in obese and elderly Korean women. Iran J Public Health. 2018;47(Suppl 1):74-81.

34. Licht CM, de Geus EJ, van Dyck R, Penninx BW. Association between anxiety disorders and heart rate variability in The Netherlands Study of Depression and Anxiety (NESDA). Psychosom Med. 2009;71(5):508-518. doi:10.1097/PSY.0b013e3181a292a6

35. Kim SW, Jung WS, Kim JW, Nam SS, Park HY. Aerobic continuous and interval training under hypoxia enhances endurance exercise performance with hemodynamic and autonomic nervous system function in amateur male swimmers. Int J Environ Res Public Health. 2021;18(8):3944. doi:10.3390/ijerph18083944

36. Qiu S, Cai X, Yin H, et al. Exercise training and endothelial function in patients with type 2 diabetes: a meta-analysis. Cardiovasc Diabetol. 2018;17(1):64. doi:10.1186/s12933-018-0711-2

37. Muangritdech N, Hamlin MJ, Sawanyawisuth K, et al. Hypoxic training improves blood pressure, nitric oxide and hypoxia-inducible factor-1 alpha in hypertensive patients. Eur J Appl Physiol. 2020;120(8):1815-1826. doi:10.1007/s00421-020-04410-9

38. Nishiwaki M, Kawakami R, Saito K, Tamaki H, Takekura H, Ogita F. Vascular adaptations to hypobaric hypoxic training in postmenopausal women. J Physiol Sci. 2011;61(2):83-91. doi:10.1007/s12576-010-0126-7

39. Kodama S, Saito K, Tanaka S, et al. Cardiorespiratory fitness as a quantitative predictor of all-cause mortality and cardiovascular events in healthy men and women: a meta-analysis. JAMA. 2009;301(19):2024-2035. doi:10.1001/jama.2009.681

40. Park HY, Jung WS, Kim J, Hwang H, Lim K. Twelve weeks of aerobic exercise at the lactate threshold improves autonomic nervous system function, body composition, and aerobic performance in women with obesity. J Obes Metab Syndr. 2020;29(1):67-75. doi:10.7570/jomes19063

41. Millet GP, Roels B, Schmitt L, Woorons X, Richalet JP. Combining hypoxic methods for peak performance. Sports Med. 2010;40(1):1-25. doi:10.2165/11317920-000000000-00000

42. Park HY, Hwang H, Park J, Lee S, Lim K. The effects of altitude/hypoxic training on oxygen delivery capacity of the blood and aerobic exercise capacity in elite athletes - a meta-analysis. J Exerc Nutr Biochem. 2016;20(1):15-22. doi:10.20463/jenb.2016.03.20.1.3

\section{Publish your work in this journal}

The International Journal of Women's Health is an international, peer-reviewed open-access journal publishing original research, reports, editorials, reviews and commentaries on all aspects of women's healthcare including gynecology, obstetrics, and breast cancer. The manuscript management system is completely online and includes a very quick and fair peer-review system, which is all easy to use. Visit http://www. dovepress.com/testimonials.php to read real quotes from published authors.

Submit your manuscript here: https://www.dovepress.com/international-journal-of-womens-health-journal 